\title{
A Higher-dimensional Origin of the Inverted Mass Hierarchy for Neutrinos
}

\author{
M. Tanimoto ${ }^{1}$ and T. Yanagida ${ }^{2}$ \\ ${ }^{1}$ Department of Physics, Niigata University, Niigata 950-2181, Japan \\ ${ }^{2}$ Department of Physics, University of Tokyo, Tokyo 113 0033, Japan
}

\begin{abstract}
We present successful lepton mass matrices with an inverted mass hierarchy for neutrinos, which follow from a geometrical structure of a $(1+5)$ dimensional space-time where two extra dimensions are compactified on the $\mathbf{T}^{\mathbf{2}} / \mathbf{Z}_{\mathbf{3}}$ orbifold. A $\mathbf{5}^{*}$ and a right-handed neutrino $N$ in each family are localized on each of the equivalent three fixed points of the orbifold while three 10's and Higgs doublets $H_{u}$ and $H_{d}$ live in the bulk. An $S_{3}$ family symmetry is assumed on three $5^{*}$ s and on three $N^{\prime}$ 's, since the three fixed points are equivalent to one another. The Higgs field $\phi$ responsible for the B-L breaking is localized on one of the three fixed points, which generates the inverted hierarchy for the neutrino masses. The baryon asymmetry is well explained in the non-thermal leptogenesis via inflaton decay. We emphasize that the present model predicts the effective neutrino mass, $\langle m\rangle_{e e}$, responsible for neutrinoless double beta decays as $\langle m\rangle_{e e} \simeq 50 \mathrm{meV}$. This will be accessible to future experiments.
\end{abstract}




\section{Introduction}

It is a challenging and important task to find an origin of the observed hierarchies in mass matrices for quarks and leptons, since it may provide us with a clue to a more fundamental theory beyond the standard model. The most remarkable observation is that the weak mixing angles in the lepton sector are of the order 1 while those in the quark sector are small. This indicates that nature of left-handed lepton doublets is very different from that of left-handed quark doublets, which means that there is a big disparity between multiplets $5^{*}$ 's and 10's in the $\mathrm{SU}(5)$ grand unification model.

It has been pointed out [1] that a $(1+5)$ dimensional space-time may explain the above disparity if extra two dimensions are compactified on the $\mathbf{T}^{\mathbf{2}} / \mathbf{Z}_{\mathbf{3}}$ orbifold. This orbifold has three equivalent fixed points. Here, a $5^{*}$ and a right-handed neutrino $N$ in each family are localized on each of the three fixed points of the orbifold, while three 10's live in the bulk. One may obtain naturally successful democratic mass matrices for quarks and leptons, provided that the three 10's, Higgs doublets and a Higgs $\phi$ responsible for the B-L symmetry breaking are distributed homogeneously in the bulk. Thus, this theory may provide a model for the almost degenerate neutrino masses.

It has been, furthermore, shown [2] that an extension of the model to the type IIB string theory may explain not only the above geometrical structure of the wave functions for $5^{*}$ 's, $N$ 's and 10's but also the presence of Higgs doublets $H_{u}$ and $H_{d}$ in the bulk. However, the nature of the B-L breaking is not clearly understood. It is remarkable that if the Higgs $\phi$ for the B-L breaking is localized on one of the three fixed points, one of $N$ acquires a superheavy Majorana mass generating an inverted hierarchy in the neutrino mass spectrum. The purpose of the present paper is to discuss this possibility.

Our analysis shows that the present model is consistent with all observations on neutrino masses and mixing angles. Further we show that the matrix element responsible for neutrinoless double beta decays is predicted as $\langle m\rangle_{e e} \simeq 50 \mathrm{meV}$. The prediction will be 
tested in future experiments.

In section 2, we discuss the neutrino and charged lepton mass matrices, where the inverted neutrino mass spectrum is naturally obtained. In section 3, the non-thermal leptogenesis via inflaton decay is discussed in the present model. The summary is devoted to section 4 .

\section{Neutrino Masses and Mixings}

Our discussion is based on the model proposed in $[1,2]$. Here, a $\mathbf{5}^{*}$ and a right-handed neutrino $N$ in each family are localized on one of the equivalent three fixed points of the $\mathbf{T}^{\mathbf{2}} / \mathbf{Z}_{\mathbf{3}}$ orbifold while three 10 's and Higgs doublets $H_{u}$ and $H_{d}$ live in the bulk. We assume in this paper that the $\phi$ for the B-L breaking is localized on the fixed point on which the $5^{*}$ and $N$ in the third family reside. Thus, the $N_{3}$ in the third family has a very large Majorana mass compared with those of other $N$ 's.

We consider an interchange symmetry between the fields on the orbifold fixed points, since the three fixed points are equivalent to one another. That is, we assume an $S_{3}$ family symmetry acting on three $5^{*}$ 's and on three $N^{\prime}$ s. Then, the neutrino and charged lepton mass matrices are given in terms of a few parameters.

However, we have to introduce breakings of the $S_{3}$ symmetry to obtain realistic mass matrices. We assume two sources of the breaking. One is a localization of the wave function of the $\phi$ and the other is small distortions of the wave functions of the three 10's and the doublet Higgses from homogeneous forms in the bulk.

\subsection{Neutrino Mass Matrix}

Let us begin by discussing the Dirac neutrino mass matrix, which is given by the Yukawa coupling matrix of $N 5^{*} H_{u}$. There are two independent matrices which are invariant of 
the $S_{3}$ symmetry $[3,4]$. Namely, the $S_{3}$ invariant Dirac mass matrix is given by [3]

$$
h_{\nu}=h_{0}\left[\left(\begin{array}{lll}
1 & 0 & 0 \\
0 & 1 & 0 \\
0 & 0 & 1
\end{array}\right)+\left(\begin{array}{ccc}
0 & \epsilon & \epsilon \\
\epsilon & 0 & \epsilon \\
\epsilon & \epsilon & 0
\end{array}\right)\right]
$$

Notice that the parameter $\epsilon$ is suppressed by separation of the three fixed points. We expect $\epsilon \simeq \exp \left(-\ell_{*} \times M_{*}\right)$ where $\ell_{*}$ and $M_{*}$ are the distance between different fixed points and a mass scale of the fundamental theory, respectively [5]. For instance, we have $\epsilon \simeq 0.001$ for $\ell_{*} \times M_{*} \simeq 7$.

We have assumed, so far, a homogeneous distribution of the Higgs fields $H_{u}$ in the bulk. A small distortion of the Higgs wave function may induce a violation of the $S_{3}$ symmetry. The breaking effects appear first in the diagonal elements of the above mass matrix and the effects in the $\epsilon$ term may be negligible for our discussion. Therefore, the neutrino Dirac mass matrix is given by

$$
h_{\nu}=h_{0}\left[\left(\begin{array}{ccc}
1 & 0 & 0 \\
0 & 1+\delta_{1} & 0 \\
0 & 0 & 1+\delta_{2}
\end{array}\right)+\left(\begin{array}{ccc}
0 & \epsilon & \epsilon \\
\epsilon & 0 & \epsilon \\
\epsilon & \epsilon & 0
\end{array}\right)\right]=h_{0}\left(\begin{array}{ccc}
1 & \epsilon & \epsilon \\
\epsilon & 1+\delta_{1} & \epsilon \\
\epsilon & \epsilon & 1+\delta_{2}
\end{array}\right) .
$$

Here, we take a basis where all the diagonal elements are real while the off diagonal elements $\epsilon$ are complex.

The Majorana mass matrix $M_{R}$ for the right-handed neutrino $N_{i}(i=1-3)$ is determined also by localization properties of $N_{i}$ and $\phi$ fields. Since the $\phi$ is assumed to reside on one of the three fixed points where the third family $N_{3}$ is localized, the $(3,3)$ element of the Majorana mass matrix dominates over other elements. Then, the Majorana mass matrix is given in terms of three independent suppression factors $\epsilon_{1}, \epsilon_{2}$ and $\epsilon_{3}$ as follows:

$$
M_{R}=M_{0}\left(\begin{array}{ccc}
\epsilon_{2} & \epsilon_{3}^{2} & \epsilon_{1} \\
\epsilon_{3}^{2} & \epsilon_{2} & \epsilon_{1} \\
\epsilon_{1} & \epsilon_{1} & 1
\end{array}\right)
$$

where we always take the three diagonal elements to be real. In the following discussion we assume all elements in the matrix $M_{R}$ to be real, for simplicity. Notice that the suppression factors come from the separation of the distinct fixed points and hence the parameters $\epsilon_{i}(i=1,2,3)$ are the same order of the magnitude of $|\epsilon|$. 
Through the seesaw mechanism $[6,7]$, the effective neutrino mass matrix is given, in the leading order of $\epsilon, \epsilon_{i}$ and $\delta_{i}$, by

$$
M_{\nu}=h_{\nu}^{T} M_{R}^{-1} h_{\nu}=\frac{h_{0}^{2}}{\epsilon_{2} M_{0}}\left(\begin{array}{ccc}
1-\frac{\epsilon_{1}^{2}}{\epsilon_{2}} & 2 \epsilon+\tilde{\epsilon} & \epsilon-\epsilon_{1} \\
2 \epsilon+\tilde{\epsilon} & 1+2 \delta_{1}-\frac{\epsilon_{1}^{2}}{\epsilon_{2}} & \epsilon-\epsilon_{1} \\
\epsilon-\epsilon_{1} & \epsilon-\epsilon_{1} & \epsilon_{2}
\end{array}\right)
$$

where $^{1}$

$$
\epsilon=|\epsilon| e^{i \varphi}, \quad \tilde{\epsilon}=\frac{\epsilon_{1}^{2}-\epsilon_{3}^{2}}{\epsilon_{2}}
$$

The neutrino mass eigenvalues are obtained as

$$
m_{1}^{2} \simeq \frac{h_{0}^{2}}{\epsilon_{2}^{2} M_{0}^{2}}, \quad m_{2}^{2} \simeq \frac{h_{0}^{2}}{\epsilon_{2}^{2} M_{0}^{2}}\left(1+4 \delta_{1}-4 \frac{\epsilon_{1}^{2}}{\epsilon_{2}}\right), \quad m_{3}^{2} \simeq \frac{h_{0}^{2}}{\epsilon_{2}^{2} M_{0}^{2}} \epsilon_{2}^{2}
$$

which are the spectrum called as inverted mass hierarchy. Therefore, the ratio of the solar neutrino mass scale and the atmospheric neutrino mass scale is given in terms of $\delta_{1}$ and $\epsilon_{i}$

$$
\frac{\Delta m_{\mathrm{solar}}^{2}}{\Delta m_{\mathrm{atm}}^{2}}=\frac{m_{2}^{2}-m_{1}^{2}}{m_{1}^{2}-m_{3}^{2}} \simeq 4\left(\delta_{1}-\frac{\epsilon_{3}^{2}}{\epsilon_{2}}\right) .
$$

The experimental data of $\Delta m_{\mathrm{solar}}^{2} \simeq 8 \times 10^{-5} \mathrm{eV}^{2}$ and $\Delta m_{\mathrm{atm}}^{2} \simeq 2.2 \times 10^{-3} \mathrm{eV}^{2}[8,9,10]$ gives us a constraint,

$$
\delta_{1} \simeq 9 \times 10^{-3}
$$

where $\epsilon_{i}$ are neglected against $\delta_{1}$. The condition $\epsilon_{i} \ll \delta_{1}$ is required to get the observed $\sin \theta_{12}$ in eq. (17) of the subsection 2.3 .

The unitary matrix, which diagonalizes the neutrino mass matrix of eq.(4) ( $V_{\nu}^{T} M_{\nu} V_{\nu}=$ $M_{\text {diagonal }}$ ), is given approximately by

$$
V_{\nu} \simeq\left(\begin{array}{ccc}
1 & \frac{2|\epsilon| \cos \varphi+\tilde{\epsilon}}{2 \delta_{1}} & \mathcal{O}\left(\epsilon, \epsilon_{i}\right) \\
-\frac{2|\epsilon| \cos \varphi+\tilde{\epsilon}}{2 \delta_{1}} & 1 & \mathcal{O}\left(\epsilon, \epsilon_{i}\right) \\
\mathcal{O}\left(\epsilon, \epsilon_{i}\right) & \mathcal{O}\left(\epsilon, \epsilon_{i}\right) & 1
\end{array}\right)
$$

It should be noticed that the $\mathrm{CP}$ violating phase $\varphi$ appears here at the order of $\epsilon$. The Majorana phases are also estimated from the mass matrix of eq.(4), where the diagonal

\footnotetext{
${ }^{1} \mathrm{~A}$ general analysis including other possible phases is prepared for $\mathrm{CP}$ violating phenomena at low energies.
} 
elements are real and off diagonal ones have imaginary parts of the order of $\epsilon$ in the unit of $h_{0}^{2} /\left(\epsilon_{2} M_{0}\right)$. After diagonalizing the mass matrix, the first and second masses $m_{1}$ and $m_{2}$ get phases of the order of $\epsilon$ while $m_{3}$ has a phase of the order of $\epsilon^{2}$. Therefore, the Majorana phases are at most of the order of $\epsilon$, which are very small.

\subsection{Charged Lepton Sector}

When the three 10's and the Higgs multiplet $H_{d}$ distribute homogeneously in the bulk, one obtains the following democratic mass matrix for charged leptons:

$$
M_{\ell}=\frac{m_{0}}{a+b+c}\left(\begin{array}{lll}
a & a & a \\
b & b & b \\
c & c & c
\end{array}\right) .
$$

In this mass matrix, the left-handed mixing is independent of values $a, b$ and $c$ while the right-handed one depends on these values. We take $a=b=c=1$, for simplicity, since our concern is the left-handed mixing angles.

Now we introduce distortions of wave functions of the 10's in the bulk. We assume that dominant effects appear on the diagonal elements of the above matrix. Then, the charged lepton mass matrix is given by ,

$$
M_{\ell}=\frac{m_{0}}{3}\left(\begin{array}{lll}
1 & 1 & 1 \\
1 & 1 & 1 \\
1 & 1 & 1
\end{array}\right)+\left(\begin{array}{ccc}
\delta_{\ell 1} & 0 & 0 \\
0 & \delta_{\ell 2} & 0 \\
0 & 0 & \delta_{\ell 3}
\end{array}\right),
$$

where the second term of the right-handed side is the $S_{3}$ breaking terms coming from the distortions of the $\mathbf{1 0}$ fields. We take all $\delta_{\ell i}$ to be real, for simplicity. This form of the mass matrix is used in ref.[11,3]. Here, we have assumed that the effects of distortion of the Higgs field $H_{d}$ are negligibly small.

The matrix of eq.(11) is diagonalized by $V_{\ell}=F_{0} L_{\ell}$, where

$$
\begin{aligned}
& F_{0}=\left(\begin{array}{ccc}
\frac{1}{\sqrt{2}} & \frac{1}{\sqrt{6}} & \frac{1}{\sqrt{3}} \\
-\frac{1}{\sqrt{2}} & \frac{1}{\sqrt{6}} & \frac{1}{\sqrt{3}} \\
0 & -\frac{2}{\sqrt{6}} & \frac{1}{\sqrt{3}}
\end{array}\right), \\
& F_{\ell} \simeq\left(\begin{array}{ccc}
\cos \theta_{\ell} & \sin \theta_{\ell} & \lambda_{\ell} \sin 2 \theta_{\ell} \\
-\sin \theta_{\ell} & \cos \theta_{\ell} & -\lambda_{\ell} \cos 2 \theta_{\ell} \\
-\lambda_{\ell} \sin 3 \theta_{\ell} & \lambda_{\ell} \cos 3 \theta_{\ell} & 1
\end{array}\right),
\end{aligned}
$$


with

$$
\begin{aligned}
& \tan 2 \theta_{\ell} \simeq \sqrt{3} \frac{\delta_{\ell 2}-\delta_{\ell 1}}{2 \delta_{\ell 3}-\delta_{\ell 2}-\delta_{\ell 1}}, \quad \lambda_{\ell} \simeq \frac{1}{3 \sqrt{2}} \frac{\xi_{\ell}}{m_{0}}, \\
& \xi_{\ell}=\sqrt{\left(2 \delta_{\ell 3}-\delta_{\ell 2}-\delta_{\ell 1}\right)^{2}+3\left(\delta_{\ell 2}-\delta_{\ell 1}\right)^{2}} .
\end{aligned}
$$

The mass eigenvalues are given by

$$
\begin{aligned}
& m_{e}=\frac{1}{3}\left(\delta_{\ell 1}+\delta_{\ell 2}+\delta_{\ell 3}\right)-\frac{1}{6} \xi_{\ell}, \\
& m_{\mu}=\frac{1}{3}\left(\delta_{\ell 1}+\delta_{\ell 2}+\delta_{\ell 3}\right)+\frac{1}{6} \xi_{\ell}, \\
& m_{\tau}=m_{0}+\frac{1}{3}\left(\delta_{\ell 1}+\delta_{\ell 2}+\delta_{\ell 3}\right) .
\end{aligned}
$$

If we take $\delta_{\ell 1}+\delta_{\ell 2}=0$ and $\delta_{\ell 2} \ll \delta_{\ell 3}$, for simplicity [3], we get following mixings in terms of the charged lepton masses:

$$
\sin \theta_{\ell} \simeq-\sqrt{\frac{m_{e}}{m_{\mu}}}, \quad \lambda_{\ell} \simeq \frac{1}{\sqrt{2}} \frac{m_{\mu}}{m_{\tau}},
$$

which is used in our numerical calculations. It may be important to note that the condition $\delta_{\ell 1}+\delta_{\ell 2}=0$ is crucial for the prediction of $\sin \theta_{\ell}$. For example, if we take another condition $\delta_{\ell 1} \ll \delta_{\ell 2} \ll \delta_{\ell 3}$, we have $\sin \theta_{\ell} \simeq-m_{e} / \sqrt{3} m_{\mu}$, which is much smaller than the one in eq.(15). Therefore, the prediction of $\sin \theta_{13}$ strongly depends on the assumption of $\delta_{\ell i}$ as seen in eq.(17).

\subsection{Lepton Flavor Mixings}

The lepton flavor mixing matrix $U$ [12] is obtained as

$$
U=F_{\ell}^{\dagger} F_{0}^{\dagger} V_{\nu},
$$

which gives

$$
\begin{aligned}
& \sin \theta_{12} \simeq-\frac{1}{\sqrt{2}}+\sqrt{\frac{m_{e}}{m_{\mu}}}+\frac{1}{\sqrt{2}} \frac{2|\epsilon| \cos \phi+\tilde{\epsilon}}{2 \delta_{1}}, \\
& \sin \theta_{13} \simeq \frac{2}{\sqrt{3}} \sqrt{\frac{m_{e}}{m_{\mu}}} \\
& \sin \theta_{23} \simeq-\frac{2}{\sqrt{3}}+\frac{1}{\sqrt{6}} \frac{m_{\mu}}{m_{\tau}}
\end{aligned}
$$


where $\theta_{i j}$ correspond to the mixing angles in the conventional parameterization of the mixing matrix in PDG [13]. For example, we take a constraint in eq.(8), $\delta_{1}=0.009$, with $|\epsilon|=0.002$ and $|\varphi|=\pi / 4$, which leads to

$$
\sin ^{2} \theta_{12}=0.30, \quad \sin ^{2} 2 \theta_{23}=0.93, \quad \sin ^{2} \theta_{13}=0.0025
$$

Those are consistent with the result of three flavor analyses on the experimental data with $3 \sigma$ in $[14,15]$,

$$
\begin{aligned}
& 7.2 \times 10^{-5} \mathrm{eV}^{2} \leq \Delta \mathrm{m}_{12}^{2} \leq 9.1 \times 10^{-5} \mathrm{eV}^{2}, \quad 0.23 \leq \sin ^{2} \theta_{12} \leq 0.38 \\
& 1.4 \times 10^{-3} \mathrm{eV}^{2} \leq \Delta \mathrm{m}_{13}^{2} \leq 3.3 \times 10^{-3} \mathrm{eV}^{2}, \quad \sin ^{2} 2 \theta_{23} \geq 0.90 \\
& \sin ^{2} \theta_{13} \leq 0.047
\end{aligned}
$$

We can also discuss the neutrinoless double beta decay rate, which is determined by an effective Majorana mass:

$$
\langle m\rangle_{e e}=\left|m_{1} c_{12}^{2} c_{13}^{2} e^{i \rho}+m_{2} s_{12}^{2} c_{13}^{2} e^{i \sigma}+m_{3} s_{13}^{2} e^{-2 i \delta_{D}}\right|
$$

where $c_{i j}$ and $s_{i j}$ denote $\cos \theta_{i j}$ and $\sin \theta_{i j}$, respectively, $\delta_{D}$ is a so called Dirac phase, and

$\rho, \sigma$ are Majorana phases. Because of $\rho, \sigma \ll 1$ as discussed after eq.(9) and $m_{1} \simeq m_{2}$, the predicted $\langle m\rangle_{e e}$ is

$$
\langle m\rangle_{e e} \simeq m_{1} \simeq 50 \mathrm{meV}
$$

which will be tested in future experiments.

\subsection{Right-handed Majorana Neutrino Mass Matrix}

Let us examine the right-handed Majorana neutrino mass matrix to discuss the leptogenesis [16]. The mass eigenvalues of $M_{R}$ in eq.(3) are obtained as

$$
m_{R 1} \simeq M_{0}\left(\epsilon_{2}-2 \epsilon_{1}^{2}+\epsilon_{3}^{2}-2 \epsilon_{1}^{2} \epsilon_{2}\right), \quad m_{R 2} \simeq M_{0}\left(\epsilon_{2}-\epsilon_{3}^{2}\right), \quad m_{R 3} \simeq M_{0}\left(1+2 \epsilon_{1}^{2}+2 \epsilon_{1}^{2} \epsilon_{2}\right)
$$

where the right-handed Majorana neutrinos of the first and second family are almost degenerated. The mass difference of $m_{R 1}$ and $m_{R 2}$ is given by

$$
m_{R 2}-m_{R 1} \simeq 2 M_{0}\left[\epsilon_{1}^{2}\left(1+\epsilon_{2}\right)-\epsilon_{3}^{2}\right]
$$


which is expected to be $\mathcal{O}\left(M_{0} \epsilon^{2} \sim M_{0} \epsilon^{3}\right)$ depending on the ratio $\epsilon_{1} / \epsilon_{3}$.

The orthogonal matrix $O_{R}$, which diagonalizes the matrix $M_{R}$, is given by

$$
O_{R} \simeq\left(\begin{array}{ccc}
\frac{1}{\sqrt{2}}+\epsilon_{x} & \frac{1}{\sqrt{2}} & -\epsilon_{1} \\
-\left(\frac{1}{\sqrt{2}}+\epsilon_{x}\right) & \frac{1}{\sqrt{2}} & \epsilon_{1} \\
\epsilon_{1} & 0 & 1
\end{array}\right), \quad \text { with } \quad O_{R}^{T} M_{R} O_{R}=M_{R}^{\text {diagonal }}
$$

where $\epsilon_{x}$ is expressed in terms of $\epsilon_{i}$.

In order to discuss the leptogenesis in the next section, we take the diagonal basis of the right-handed Majorana neutrino mass matrix. Then, the Dirac neutrino mass matrix $h_{\nu}$ is converted to $O_{R}^{T} h_{\nu}$. However, for our convenience, we use $\bar{h}_{\nu}=O_{R}^{T} h_{\nu} O_{R}$ instead of $O_{R}^{T} h_{\nu}:$

$$
\bar{h}_{\nu} \simeq h_{0}\left(\begin{array}{ccc}
1+\frac{\delta_{1}}{2}-|\epsilon| e^{i \varphi}+2 \sqrt{2} \epsilon_{x} & -\frac{\delta_{1}}{2} & (1-\sqrt{2}) \epsilon_{1} \\
-\frac{\delta_{1}}{2} & 1+\frac{\delta_{1}}{2}+|\epsilon| e^{i \varphi} & \sqrt{2}|\epsilon| e^{i \varphi} \\
(1-\sqrt{2}) \epsilon_{1} & \sqrt{2}|\epsilon| e^{i \varphi} & 1+\delta_{2}
\end{array}\right)
$$

The product of $\bar{h}_{\nu} \bar{h}_{\nu}^{\dagger}$ appears in the calculations of the leptogenesis.

\section{$3 \quad$ Leptogenesis}

We consider the leptogenesis $[16,17]$ via decays of the right-handed neutrinos $N_{R i}$ which are produced non-thermally by decays of the inflaton $\varphi_{\text {inf }}$ [18].

If the phase $\varphi$ does not vanish, CP invariance is vaiolated in the Yukawa matrix $h_{\nu}$. Then, the interference between decay amplitudes of tree and one-loop diagrams results in the lepton number production [16]. The lepton number asymmetry per decay of the right-handed neutrino $N_{R i}$ is given by $[16,19]$

$$
\begin{aligned}
\epsilon_{\ell i} & \equiv \frac{\sum_{j} \Gamma\left(N_{R i} \rightarrow l_{L j}+H\right)-\sum_{j} \Gamma\left(N_{R i} \rightarrow \overline{l_{L j}}+\bar{H}\right)}{\sum_{j} \Gamma\left(N_{R i} \rightarrow l_{L j}+H\right)+\sum_{j} \Gamma\left(N_{R i} \rightarrow \overline{l_{L j}}+\bar{H}\right)} \\
& =-\frac{1}{8 \pi} \frac{1}{\left(\bar{h}_{\nu} \bar{h}_{\nu}^{\dagger}\right)_{i i}} \sum_{k \neq i} \operatorname{Im}\left[\left\{\left(\bar{h}_{\nu} \bar{h}_{\nu}^{\dagger}\right)_{i k}\right\}^{2}\right]\left[\mathcal{F}_{V}\left(\frac{M_{k}^{2}}{M_{i}^{2}}\right)+\mathcal{F}_{S}\left(\frac{M_{k}^{2}}{M_{i}^{2}}\right)\right]
\end{aligned}
$$

where $N_{R i}, l_{L j}$, and $H\left(\overline{l_{L j}}\right.$ and $\left.\bar{H}\right)$ symbolically denote fermionic or scalar components of corresponding supermultiplets (and their anti-particles), and $\mathcal{F}_{V}(x)$ and $\mathcal{F}_{S}(x)$ represent contributions from vertex and self-energy diagrams, respectively. 
In the case of the SUSY theory, they are given by [20]

$$
\mathcal{F}_{V}(x)=\sqrt{x} \ln \left(1+\frac{1}{x}\right), \quad \mathcal{F}_{S}(x)=\frac{2 \sqrt{x}}{x-1}
$$

Here, we have assumed that the mass difference of the right-handed neutrinos is large enough compared with their decay widths so that the perturbative calculation is ensured.

The relevant Yukawa couplings are given as

$$
\begin{aligned}
& \operatorname{Im}\left[\left\{\left(\bar{h}_{\nu} \bar{h}_{\nu}^{\dagger}\right)_{12}\right\}^{2}\right]=-\operatorname{Im}\left[\left\{\left(\bar{h}_{\nu} \bar{h}_{\nu}^{\dagger}\right)_{21}\right\}^{2}\right]=-2\left|h_{0}\right|^{4}|\epsilon| \delta_{1}^{2}\left[1+(2-\sqrt{2}) \frac{\epsilon_{1}}{\delta_{1}}\right] \sin \varphi, \\
& \operatorname{Im}\left[\left\{\left(\bar{h}_{\nu} \bar{h}_{\nu}^{\dagger}\right)_{13}\right\}^{2}\right]=2\left|h_{0}\right|^{4}|\epsilon| \epsilon_{1}\left[2(2 \sqrt{2}-3) \epsilon_{1}-(2-\sqrt{2}) \delta_{1}\right] \sin \varphi \\
& \operatorname{Im}\left[\left\{\left(\bar{h}_{\nu} \bar{h}_{\nu}^{\dagger}\right)_{23}\right\}^{2}\right]=2\left|h_{0}\right|^{4}|\epsilon|^{2}\left(2 \delta_{2}-\delta_{1}\right) \sin 2 \varphi, \\
& \left(\bar{h}_{\nu} \bar{h}_{\nu}^{\dagger}\right)_{11}=\left|h_{0}\right|^{2}\left(1+\delta_{1}-2|\epsilon| \cos \varphi\right), \\
& \left(\bar{h}_{\nu} \bar{h}_{\nu}^{\dagger}\right)_{22}=\left|h_{0}\right|^{2}\left(1+\delta_{1}+2|\epsilon| \cos \varphi\right) .
\end{aligned}
$$

Since the right-handed neutrino masses of the first and second families are almost degenerate in the present model ${ }^{2}$, the self-energy contribution $\mathcal{F}_{S}(x)$ is much larger than the vertex contribution $\mathcal{F}_{V}(x)$ as follows:

$$
\begin{aligned}
& \mathcal{F}_{V}\left(\frac{m_{R 2}^{2}}{m_{R 1}^{2}}\right) \simeq \mathcal{F}_{V}\left(\frac{m_{R 1}^{2}}{m_{R 2}^{2}}\right) \simeq \ln 2 \\
& \mathcal{F}_{S}\left(\frac{m_{R 2}^{2}}{m_{R 1}^{2}}\right)=-\mathcal{F}_{S}\left(\frac{m_{R 1}^{2}}{m_{R 2}^{2}}\right)=\frac{2 m_{R 1} m_{R 2}}{m_{R 2}^{2}-m_{R 1}^{2}} \simeq \frac{1}{2} \frac{\epsilon_{2}\left(\epsilon_{2}-2 \epsilon_{1}^{2}\right)}{\epsilon_{2}\left(\epsilon_{1}^{2}-\epsilon_{3}^{2}\right)+\epsilon_{1}^{2}\left(\epsilon_{2}^{2}+\epsilon_{3}^{2}-\epsilon_{1}^{2}\right)} .
\end{aligned}
$$

Therefore, we have

$$
\mathcal{F}_{S}\left(\frac{m_{R 2}^{2}}{m_{R 1}^{2}}\right)=-\mathcal{F}_{S}\left(\frac{m_{R 1}^{2}}{m_{R 2}^{2}}\right)=\mathcal{O}\left(\epsilon_{2}^{-1} \sim \epsilon_{2}^{-2}\right)
$$

depending on the ratio $\epsilon_{1} / \epsilon_{3}$. Notice that a large enhancement is obtained in the case of $\epsilon_{1}=\epsilon_{3}$.

The lepton number asymmetry is given

$$
\epsilon_{\ell 1}=\epsilon_{\ell 2} \simeq \frac{\left|h_{0}\right|^{2}}{4 \pi}|\epsilon| \delta_{1}^{2}\left(2-\sqrt{2} \frac{\epsilon_{1}}{\delta_{1}}\right) \frac{\epsilon_{2}\left(\epsilon_{2}-2 \epsilon_{1}^{2}\right)}{\epsilon_{2}\left(\epsilon_{1}^{2}-\epsilon_{3}^{2}\right)+\epsilon_{1}^{2}\left(\epsilon_{2}^{2}+\epsilon_{3}^{2}-\epsilon_{1}^{2}\right)} \sin \varphi,
$$

${ }^{2}$ The leptogenesis with almost degenerate Majorana neutrinos has been examined in the framework of the democratic mass matrix [21]. 
which turns out

$$
\epsilon_{\ell 1}=\epsilon_{\ell 2} \simeq \frac{\left|h_{0}\right|^{2}}{2 \pi} \delta_{1}^{2} \sin \varphi
$$

The value of $\left|h_{0}\right|^{2}$ is given in terms of $m_{1}$ and $m_{R 1}$ :

$$
\left|h_{0}\right|^{2} \simeq \frac{m_{1} m_{R 1}}{(174 \sin \beta \mathrm{GeV})^{2}} \simeq \frac{1.65}{\sin ^{2} \beta} \times 10^{-15} \frac{m_{R 1}}{1 \mathrm{GeV}}
$$

where the vev of the Higgs $H_{u}, 174 \sin \beta \mathrm{GeV}$, and $m_{1}=0.05 \mathrm{eV}$ are used. The ratio of the lepton number density $n_{L}$ to the entropy density $s$ produced by the inflaton decay is given by [18]

$$
\frac{n_{L}}{s}=\frac{3}{2} \sum_{i} \epsilon_{\ell i} B_{r}^{(i)} \frac{T_{R}}{m_{\phi_{i n f}}}
$$

where $T_{R}$ is the reheating temperature after the inflation, $m_{\phi_{i n f}}$ the mass of the inflaton, and $B_{r}^{(i)}$ the branching ratio of the decay channel of the inflaton to $N_{R i}$, i.e., $B_{r}^{(i)}=$ $B_{r}\left(\phi \rightarrow N_{R i} N_{R i}\right)$. Here, we have assumed that the inflaton decays into a pair of righthanded neutrinos, and $M_{R i}>T_{R}$ in order to make the generated lepton asymmetry not washed out by lepton-number violating processes after the $N_{R i}$ 's decay. A part of the produced lepton asymmetry is immediately converted [16] into the baryon asymmetry via the "sphaleron" effect [22], since the decays of $N_{R i}$ take place much before the electroweak phase transition. The baryon asymmetry is given by

$$
\frac{n_{B}}{s}=C \frac{n_{L}}{s}
$$

where $C$ is given by $C \simeq-0.35$ in the minimal SUSY standard model [23].

Therefore, the amount of the baryon asymmetry in the present model is estimated as

$$
\frac{n_{B}}{s} \simeq-\frac{0.7}{\sin ^{2} \beta} \times 10^{-6} \delta_{1}^{2} \sin \varphi\left(B_{r}^{(2)}+B_{r}^{(3)}\right)\left(\frac{T_{R}}{10^{10} \mathrm{GeV}}\right)\left(\frac{2 \mathcal{M}_{R}}{m_{\phi_{\text {inf }}}}\right)
$$

We see that the observed baryon asymmetry $n_{B} / s \simeq(0.8-0.9) \times 10^{-10}[13]$ is obtained for a choice of the parameter, $T_{R}=10^{10} \mathrm{GeV}$ and $\delta_{1}^{2}=10^{-4}$ with $\varphi=-\pi / 4$. 
We should note a possibility of enhancement of $\epsilon_{\ell 1}$ and $\epsilon_{\ell 2}$. The lepton number asymmetries in the eq.(31) are enhanced in the case of $\epsilon_{1}=\epsilon_{3}$, where we obtain

$$
\epsilon_{\ell 1}=\epsilon_{\ell 2} \simeq \frac{\left|h_{0}\right|^{2}}{2 \pi} \frac{\delta_{1}^{2}}{|\epsilon|} \sin \varphi
$$

where $\epsilon_{2}=\epsilon_{1}=|\epsilon|$ is taken. Therefore, the baryon asymmetry $n_{B} / s$ is enhanced by the order of $10^{3}$ because of $|\epsilon| \simeq 10^{-3}$. Then, $T_{R}=10^{7} \mathrm{GeV}$ is allowed to reproduce the observed baryon asymmetry.

\section{Summary}

We have discussed the neutrino mass matrix in a $(1+5)$ dimensional space-time where two extra dimensions are compactified on a $\mathbf{T}^{\mathbf{2}} / \mathbf{Z}_{\mathbf{3}}$ orbifold $[1,2]$. Here, a $\mathbf{5}^{*}$ and a right-handed neutrino $N$ in each family are localized on each of the equivalent three fixed points of the $\mathbf{T}^{\mathbf{2}} / \mathbf{Z}_{\mathbf{3}}$ orbifold while three 10's and Higgs doublets $H_{u}$ and $H_{d}$ live in the bulk. We have considered, in this paper, that the Higgs field $\phi$ responsible for the B-L breaking is localized on the fixed point of the third family of $5^{*}$ and $N$. This setting leads to a hierarchy in the Majorana mass spectrum for the right-handed neutrinos, $m_{R 1} \simeq m_{R 2} \ll m_{R 3}$, which naturally generates the inverted hierarchy of the neutrino masses.

The present model explains the deviation of the $\theta_{12}$ from the maximal mixing. It also predicts the element of the neutrino mass matrix, $\langle m\rangle_{e e}$, responsible for neutrinoless double beta decays as $\langle m\rangle_{e e} \simeq 50 \mathrm{meV}$, which will be accessible to future experiments. The mixing angle $\theta_{13}$ strongly depends on $S_{3}$ breaking terms.

We have shown that the observed baryon asymmetry in the present universe is produced by the non-thermal leptogenesis via the inflaton decay. Due to $m_{R 1} \simeq m_{R 2}$ the baryon asymmetry is enhanced. In conclusion, we have found that the leptogenesis works well with the reheating temperature $T_{R}=10^{7} \sim 10^{10} \mathrm{GeV}$. 
Acknowledgement The work of M.T. has been supported by the Grant-in-Aid for Science Research of the Ministry of Education, Science, and Culture of Japan No. 16028205, No. 17540243. The work of T.Y. has been supported in part by a Humboldt Research Award.

\section{References}

[1] T. Watari and T. Yanagida, Phys. Lett. B544 (2002) 167.

[2] T. Watari and T. Yanagida, Phys. Rev. D70 (2004) 036009.

[3] M. Fukugita, M. Tanimoto and T. Yanagida, Phys. Rev. D57 (1998) 4429;

M. Tanimoto, Phys. Rev. D59 (1999) 017304.

[4] H. Fritzsch and Z. Xing, Phys. Lett. B372 (1996) 265; ibid. B440 (1998) 313.

[5] N. Arkani-Hamed and S. Dimopoulos, Phys. Rev. D65 (2002) 052003.

[6] T. Yanagida, in Proceedings of the "Workshop on the Unified Theory and the Baryon Number in the Universe", Tsukuba, Japan, 1979, edited by O. Sawada and A. Sugamoto, KEK Report No. KEK-79-18, p. 95; Prog. Theor. Phys. 64 (1980) 1103;

P. Ramond, in Talk given at the Sanibel Symposium, Palm Coast, Fla., 1979, preprint CALT-68-709;

S.L. Glashow, in Quarks and leptons, proceedings of the advanced study institute (Cargèse, Corsica, 1979), J.-L. Basdevant et al. eds., Plenum, New York 1981.

[7] For an early work, see P. Minkowski, Phys. Lett. B67 (1977) 421.

See also R.N. Mohapatra and G. Senjanovic, Phys. Rev. Lett. 44 (1980) 912.

[8] Super-Kamiokande Collaboration (S. Fukuda et al.), Phys. Rev. Lett. 86 (2001) 5651, 5656 ; 
SNO Collaboration (Q. R. Ahmad et al.), Phys. Rev. Lett. 87 (2001) 071301; ibid. 89 (2002) 011301; ibid. 89 (2002) 011302; ibid. 92 (2004) 181301.

[9] KamLAND Collaboration (K. Eguchi et al.), Phys. Rev. Lett. 90 (2003) 0212021; KamLAND Collaboration (T. Araki et al.), Phys. Rev. Lett. 94 (2005) 081801.

[10] Super-Kamiokande Collaboration (Y. Fukuda et al.), Phys. Rev. Lett. 81 (1998) 1562; ibid. 82 (1999) 2644; ibid. 82 (1999) 5194.

[11] Y. Koide, Phys. Rev. D28 (1983) 252, Phys. Rev. D39 (1989) 1391.

[12] Z. Maki, M. Nakagawa and S. Sakata, Prog. Theor. Phys. 28 (1962) 870.

[13] S. Eidelman, et al. [Particle Data Group Collaboration], Phys. Lett. B592 (2004) 1.

[14] M. Maltoni, T. Schwetz, M. Tortola and J.W.F. Valle, New J. Phys 6 (2004) 122.

[15] G.L. Fogli, E. Lisi, A. Marrone and A. Palazzo, hep-ph/0506083.

[16] M. Fukugita and T. Yanagida, Phys. Lett. B174 (1986) 45.

[17] For reviews and references, see, for example, W. Buchmuller and M. Plumacher, Int. J. Mod. Phys. A15 (2000) 5047;

W. Buchmuller, R.D. Peccei and T. Yanagida, hep-ph/0502169.

[18] K. Kumekawa, T. Moroi and T. Yanagida, Prog. Theor. Phys. 92 (1994) 437;

T. Asaka, K. Hamaguchi, M. Kawasaki and T. Yanagida, Phys. Lett. B464 (1999) 12; Phys. Rev. D61 (2000) 083512;

G. F. Giudice, M. Peloso, A. Riotto and I. Tkachev, JHEP 9908 (1999) 014.

See also G. Lazarides and Q. Shafi, Phys. Lett. B258 (1991) 305.

[19] M. Flanz, E. A. Paschos and U. Sarkar, Phys. Lett. B345 (1995) 248 [Erratum-ibid. B384 (1995) 487]; 
L. Covi, E. Roulet and F. Vissani, Phys. Lett. B384 (1996) 169;

W. Buchmuller and M. Plumacher, Phys. Lett. B431 (1998) 354.

[20] L. Covi, E. Roulet and F. Vissani, in Ref. [19].

[21] M. Fujii, K. Hamaguchi, M. Kawasaki and T. Yanagida, Phys. Rev. D65 (2002) 115012.

[22] V. A. Kuzmin, V. A. Rubakov and M. E. Shaposhnikov, Phys. Lett. B155 (1985) 36.

[23] S. Y. Khlebnikov and M. E. Shaposhnikov, Nucl. Phys. B308 (1988) 885;

J. A. Harvey and M. S. Turner, Phys. Rev. D42 (1990) 3344. 